\title{
Simple method for computing scattered radiation in breast tomosynthesis
}

\author{
Oliver Diaz ${ }^{a, b}$, Premkumar Elangovan ${ }^{c}$, Kenneth C Young ${ }^{c, d}$, Kevin \\ Wells ${ }^{a}$ and David R Dance ${ }^{c, d}$ \\ ${ }^{a}$ CVSSP, University of Surrey, Guildford GU2 7XH, United Kingdom \\ ${ }^{b}$ VICOROB, University of Girona, Girona, 17071, Spain \\ ${ }^{c}$ NCCPM, Royal Surrey County Hospital, Guildford, GU2 7XX, United Kingdom \\ ${ }^{d}$ Department of Physics, University of Surrey, Guildford, GU2 7XH, United Kingdom
}

Corresponding author: Oliver Diaz (oliver.diaz@udg.edu)

\begin{abstract}
Purpose: Virtual clinical trials (VCT) are a powerful imaging tool that can be used to investigate digital breast tomosynthesis (DBT) technology. In this work, a fast and simple method is proposed to estimate the $2 \mathrm{D}$ distribution of scattered radiation which is needed when simulating DBT geometries in VCTs.

Methods: Monte Carlo simulations are used to pre-calculate scatter-to-primary ratio (SPR) for a range of low-resolution homogeneous phantoms. The resulting values can be used to estimate the 2D-distribution of scattered radiation arising from inhomogeneous anthropomorphic phantoms used in VCTs. The method has been validated by comparing the values of the scatter thus obtained against the results of direct Monte Carlo simulation for three different types of inhomogeneous anthropomorphic phantoms.
\end{abstract}

Results: Differences between the proposed scatter field estimation method and the ground truth data for the OPTIMAM phantom had an average modulus and standard deviation of over the projected breast area of $2.4 \pm 0.9 \%$ (minimum $-17.0 \%$, maximum $27.7 \%$ ). The corresponding values for the University of Pennsylvania and Duke University breast phantoms were $1.8 \pm 0.1 \%$ (minimum $-8.7 \%$, maximum $8.0 \%$ ) and 5.1 $\pm 0.1 \%$ (minimum $-16.2 \%$, maximum $7.4 \%$ ), respectively.

Conclusions: The proposed method, which has been validated using three of the most common breast models, is a useful tool for accurately estimating scattered radiation in VCT schemes used to study current designs of DBT system.

Key words: scattered radiation, SPR, Monte Carlo simulations, anthropomorphic breast models, virtual clinical trial 


\section{${ }_{35}$ I. Introduction}

Radiological images, such as mammograms and breast tomosynthesis projections, are created within image receptors using the energy deposited from primary and scattered x-ray photons. The distribution of primary x-ray photons contains useful information to visually separate the different tissue types in an x-ray image. However, scattered x-ray photons act as an undesirable signal that degrades the image quality affecting both low (e.g. masses) and high (e.g. calcifications and small fibres) frequency objects, and therefore diagnostic effectiveness is also compromised ${ }^{1,2}$. In digital systems, the scatter degradation is typically quantified using the differential signal-to-noise ratio $(\mathrm{SNRd})$ or contrast-to-noise ratio $(\mathrm{CNR})^{3,4,5}$. Such reduction in CNR due to scattered radiation has been quantified up to $54 \%$ in digital breast tomosynthesis (DBT) for a $50 \mathrm{~mm}$ thick breast ${ }^{6}$. For these reasons, scatter estimation in breast $\mathrm{x}$-ray imaging has been a topic under investigation for many years $7,8,9,10,11,12,13$.

Estimation of scattered radiation can lead to scatter signal reduction strategies in clinical environment ${ }^{14,15,16,17,18,19}$, however, it can also help in the design of image simulation frameworks by enabling the addition of the correct amount of scattered radiation in simulated image data ${ }^{20,21,22,23,24}$. Some of these frameworks are used within virtual clinical trials (VCTs) for lesion detection performance ${ }^{23,24,25,26}$. This work is concerned with fast schemes to estimate the background scatter contribution to images for use in VCTs. Since the scattered radiation can have a comparable or higher contribution to the total DBT image than the primary signal in the thickest breasts $^{11}$, it is crucial to estimate this signal properly in order to simulate realistically x-ray breast images.

Typically, conventional 2D mammography employs geometrical rejection (i.e. antiscatter grids) to reduce the amount of scattered radiation arriving at the image receptor. In this modelling situation, for a constant thickness phantom, the use of a uniform scatter signal in the central area of the image can be considered ${ }^{27}$. However, most DBT geometries do not 
include such geometrical rejection and the scatter variation across the projected breast area should be taken into account ${ }^{9,11,12,28,29,30}$. A fall of more than $50 \%$ can be observed at the chest wall, whereas the scatter signal increases considerably near the borders of the breast, mostly due to the scattered radiation arising from both the compression paddle and breast support $^{7,11,30}$. Also, the use of tilted compression paddles can vary the scattered radiation distribution $^{31}$. Therefore, scattered radiation is typically estimated via software in DBT.

Various approaches have been made to estimate the variation of the scatter across the projection images acquired in DBT. Direct Monte Carlo simulations using a realistic anthropomorphic model of the breast can be very time consuming unless considerable computing resources are available. It is therefore of interest to explore approaches using simplified models of the breast or results which have been pre-calculated. The work of Sechopoulos and colleagues used simple breast phantoms to provide data for use in scatter correction. Sechopoulos et $a^{11}$ proposed equations, for both cranial-caudal and mediolateral-oblique views, to estimate the scatter field at the centre of mass based on a curve fitting approach. These multiparameter equations depend on compressed breast thickness $(\mathrm{T})$ and projection angle $(\theta)$, and assume homogeneous breast models. Feng and Sechopoulos ${ }^{14}$ later developed a method which uses image registration algorithms to match image contours with the precalculated scatter-to-primary ratio (SPR) maps from direct Monte Carlo simulations. In an extended version, Feng et $\mathrm{l}^{15}$ reduced the computational time by generating a scatter map library from principal component analysis-based breast models. In both publications, image quality improved after the reconstruction process. Diaz et al ${ }^{12}$ suggested another accurate and fast method for scatter field estimation based on a pixel-wise image convolution which takes into account breast thickness, volumetric glandularity $(\mathrm{G})$, projection angle and breast curvature using a realistic geometry. More recently, Kim et $\mathrm{al}^{19}$ proposed an iterative scatter correction method using a fibroglandular-adipose tissue ratio model based on graphics processing unit Monte Carlo simulations, which reduce significantly the computational time. The use of such simple and fast schemes for VCTs is attractive, but the question remains 
whether scatter estimations based on simple breast models agree sufficiently well with full Monte Carlo calculations using the same anthropomorphic breast model used in a VCT.

In this work, a new fast method is proposed to accurately estimate scattered radiation for realistic DBT geometries for use in VCTs. This is achieved using normalised scatter maps from pre-calculated Monte Carlo simulations of low resolution homogeneous phantoms. The method relies on the assumption that the scattered radiation produced from such simplified phantoms approximates well the scatter generated from anthropomorphic heterogeneous breast models ${ }^{32}$. This has been tested by comparing the results with full Monte Carlo simulations using the OPTIMAM anthropomorphic breast phantom ${ }^{33}$ and then validated using two other commonly used anthropomorphic breast phantoms ${ }^{34,35}$.

\section{Materials and methods}

The spatial distribution of the SPR across the receptor plane has been calculated using Monte Carlo using breast phantoms of uniform composition and the results used to estimate the scatter distribution produced by a series of OPTIMAM anthropomorphic breast phantoms ${ }^{33}$. The method has been validated for a range of breast thicknesses, glandularities and DBT projection angles by comparison with the results of full Monte Carlo simulations of scatter produced by this series of phantoms. Further validations were then performed using two other types of anthropomorphic breast phantom.

\section{II.A. Monte Carlo simulation and geometry}

The DBT geometry of the Hologic Dimensions Selenia x-ray system (Hologic, Inc; Bedford, Massachusetts, USA) has been implemented in a Monte Carlo radiation transport package. Following the American Association of Physicists in Medicine (AAPM) Task Group \#268 
recommendations ${ }^{36}$, the details of the simulation are described below.

The simulated setup (Figure 1) included an infinitesimal point x-ray source located at a distance of $700 \mathrm{~mm}$ from the receptor plane, a $2.8 \mathrm{~mm}$ thick compression paddle $(24 \times 18$ $\mathrm{cm}^{2}$; polycarbonate with density $1.2 \mathrm{~g} / \mathrm{cm}^{3}$ ), a compressed breast model (details provided in the next section), a $1 \mathrm{~mm}$ thick breast support $\left(29 \times 24 \mathrm{~cm}^{2}\right.$; carbon fibre with density $1.8 \mathrm{~g} / \mathrm{cm}^{3}$ ), a $24 \mathrm{~mm}$ air gap between support and detector, and finally a detector corresponding to a $200 \mu \mathrm{m}$ thick layer $\left(24 \mathrm{x} 18 \mathrm{~cm}^{2}\right.$; selenium) with its corresponding cover. The x-ray beam was collimated to match the compression paddle dimension $\left(24 \times 18 \mathrm{~cm}^{2}\right)$, which was directly in contact with the top surface of the breast model. The rotation point of the DBT system was located in the middle of the detector layer and projection angles of $0,7.5$ and 25 degrees were simulated. Although the Hologic Dimensions Selenia system works typically in the \pm 7.5 degrees range, a 25 degrees projection angle was included to investigate the widest projection angles used by other DBT vendors (e.g. Siemens Mammomat Inspiration (Siemens Healthcare GmbH; Erlangen, Germany) ) ${ }^{37}$.

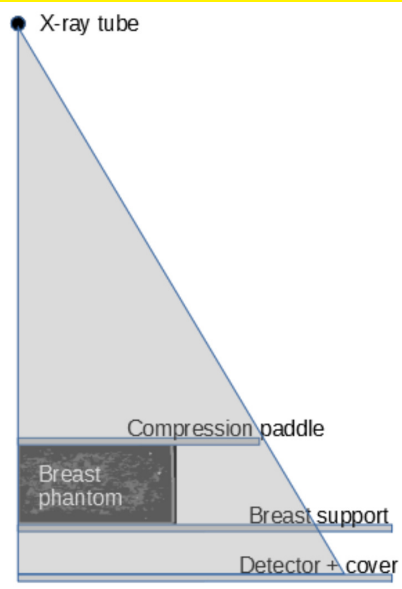

(a)

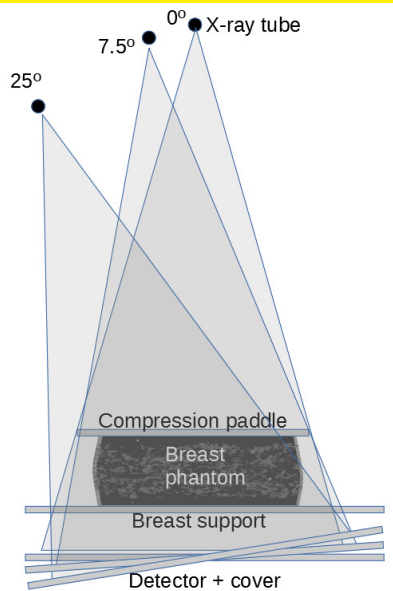

(b)

Figure 1: Schematic diagram of the geometry used in the Monte Carlo simulations in (a) sagittal and (b) coronal view. The detector tilts with projection angles. Note that the figures are not in scale.

The Monte Carlo simulation toolkit used was Geant4 (10.02.patch-02; June 2016) ${ }^{38,39}$ with the Geant 4 physics package option 4 (default particle cut-off of $1 \mathrm{~mm}$ ). This model uses 
the most accurate standard and low-energy models (e.g. atomic deexcitation) for particle simulations within the mammographic energy range. In order to speed up the simulation time, secondary particles (e.g. fluorescence, electrons, etc.) were assumed to be locally absorbed. Since the sensitive layer of the detector was made of Se (K-edge of $12.658 \mathrm{keV}$ ), these secondary particles will not travel very far at this energy, and local absorption was assumed. This has been internally tested by us and differences of $0.01 \%$ in energy deposition were observed.

This Geant4 code has been previously validated using AAPM's reference dataset ${ }^{40}$ and implemented for other scattered radiation related studies ${ }^{18,41}$. Experiments were run using sufficient events (between $15 \times 10^{9}$ to $20 \times 10^{9}$ depending on breast thickness) to produce average standard errors of the mean (SEM) below 1.5\% for primary and scatter signals for each $1 \mathrm{~mm}^{2}$ pixel within the projected breast area. The simulations were run using an Intel Xeon CPU E5-2630 v4 @ 2.20GHz and the computing times for simulation were 10-15 hours and 6-7 hours per projection for high and low resolution breast phantoms respectively, as described in the next section.

Energies deposited within the Se layer (pixel size of $1 \mathrm{~mm}^{2}$ ) by both primary $(P(x, y)$ ) and scattered $(S(x, y))$ photons from the geometry were scored independently. All photons undertaking a scatter interaction (coherent or incoherent) above the detector volume were labelled as scattered photons. Otherwise they were considered primary photons. Since the scattered radiation in the image varies slowly with position, a large pixel size was used $^{11,12}$. The resulting values of $\mathrm{S}(\mathrm{x}, \mathrm{y})$ and $\mathrm{P}(\mathrm{x}, \mathrm{y})$ were combined for each pixel to produce a $\operatorname{SPR}(x, y)$ map.

The x-ray energy spectra used were obtained from Boone et $\mathrm{al}^{42}$. Appropriate filtration was added to match the measured first half-value layer (HVL) ${ }^{43}$ and better represents the spectra found on the Hologic system. Table 1 details the beam quality used for each of the breast phantom models simulated. 


\section{II.B. Breast models}

The method was developed using ground truth data calculated using OPTIMAM heterogeneous anthropomorphic breast models (Elangovan et $\mathrm{al}^{33}$ ). These are the models we use in our VCTs ${ }^{23,44}$. The external shape of these models was extracted from thresholded DBT reconstructed volumes. The breast skin was modelled as a $1.5 \mathrm{~mm}$ thick layer and the voxel size was 500x500x500 $\mu \mathrm{m}^{3}$. Different glandularity distributions (P1, P2, P3) for a given volumetric glandularity were produced to investigate variation in the scatter field distribution under these conditions. Sample central slices of the models are shown in Figure 2(a-c).

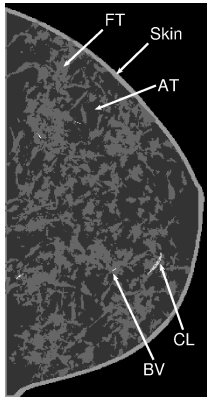

(a)

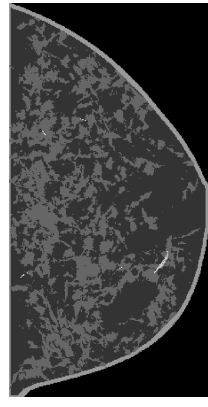

(b)

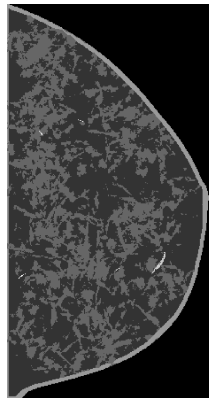

(c)

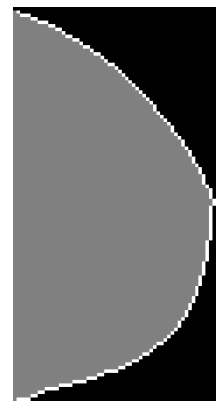

(d)

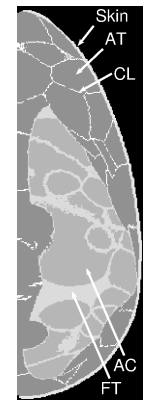

(e)

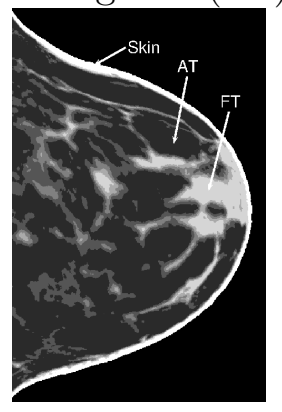

(f)

Figure 2: (a-c) Central slices of $6 \mathrm{~cm}$ thick compressed voxelised breast phantoms, $12 \mathrm{~cm}$ radius and volumetric glandularity $17 \%$ using different patterns (P1-P3). The corresponding homogeneous breast model is shown in (d). Note that the homogeneous breast has a lower resolution. Sample slices of breast models developed at UPenn ${ }^{34}$ and Duke University ${ }^{35}$ are depicted in (e) and (d), respectively. The different tissues has been indicated: Skin, fibroglandular tissue (FT), adipose tissue (AT), Cooper's ligaments (CL), blood vessels (BL) and adipose cavities (AC).

The labels associated with each voxel in the heterogeneous phantoms corresponded to: adipose tissue, fibroglandular tissue, skin, blood vessels and Cooper's ligaments. Each model also had an associated T, G and an estimated radius ( $R$ ) corresponding to the distance from the chest wall to the nipple. The thicknesses, radii and volumetric glandularities for the different breast models studied are given in Table 1. Note that for selected phantoms, more than one glandularity was investigated to analyse the effects of glandularity on the scattered radiation distribution. 
Table 1: Configuration of breast phantoms and energy spectra used in this work.

\begin{tabular}{|c|c|c|c|c|c|}
\hline Phantom & $\begin{array}{c}\mathrm{T} \\
(\mathrm{cm})\end{array}$ & $\begin{array}{c}\mathrm{R} \\
(\mathrm{cm})\end{array}$ & $\begin{array}{l}\mathrm{G} \\
(\%)\end{array}$ & $\begin{array}{l}\text { Volume } \\
\left(\mathrm{cm}^{3}\right)\end{array}$ & $\begin{array}{c}\text { Energy spectrum } \\
\left(1^{\text {st }} \mathrm{HVL}\right) \\
\end{array}$ \\
\hline \multirow{3}{*}{ Elangovan et $\mathrm{al}^{33}$} & 3 & $\begin{array}{l}4 \\
8\end{array}$ & 35 & $\begin{array}{c}73.05 \\
286.12\end{array}$ & $\begin{array}{c}28 \mathrm{kVp} \mathrm{W} / \mathrm{Al} \\
(0.49 \mathrm{~mm} \mathrm{Al})\end{array}$ \\
\hline & 6 & $\begin{array}{c}8 \\
12\end{array}$ & $\begin{array}{c}17 \\
2,17,40\end{array}$ & $\begin{array}{c}572.00 \\
1,069.74\end{array}$ & $\begin{array}{l}33 \mathrm{kVp} \mathrm{W} / \mathrm{Al} \\
(0.59 \mathrm{~mm} \mathrm{Al})\end{array}$ \\
\hline & 9 & $\begin{array}{l}12 \\
16\end{array}$ & 9 & $\begin{array}{l}1,604.51 \\
2,837.72\end{array}$ & $\begin{array}{l}42 \mathrm{kVp} \mathrm{W} / \mathrm{Al} \\
(0.73 \mathrm{~mm} \mathrm{Al})\end{array}$ \\
\hline Bakic et $\mathrm{al}^{34}$ & 5 & 5 & 20 & 225.57 & $31 \mathrm{kVp} \mathrm{W} / \mathrm{Al}(0.55 \mathrm{~mm} \mathrm{Al})$ \\
\hline Li et $\mathrm{al}^{35}$ & 6 & 9 & 32 & 539.08 & $33 \mathrm{kVp} \mathrm{W} / \mathrm{Al}(0.59 \mathrm{~mm} \mathrm{Al})$ \\
\hline
\end{tabular}

The low resolution homogeneous models used to approximate the SPR and scatter had a voxel size of $2000 \times 2000 \times 2000 \mu \mathrm{m}^{3}$ and the external shape matched to the anthropomorphic model that was being simulated. The volumes of these models were filled with uniform tissue with glandularity equivalent to the average glandularity of the heterogeneous model (Figure 2(d)). The skin voxels were not modified and therefore included in the uniform tissue.

In order to investigate the adaptability of the proposed methodology, two other breast models were investigated. First, a compressed $5 \mathrm{~cm}$ thick breast model of glandularity $20 \%$ developed at the University of Pennsylvania (UPenn $)^{34}$. This phantom had a voxel size of 500x500x500 $\mu \mathrm{m}^{3}$ and contained 6 tissue types (skin, fibroglandular and adipose tissue, ducts, cavities and ligaments). Secondly, a compressed $6 \mathrm{~cm}$ thick breast phantom developed at Duke University ${ }^{35}$ with a glandularity of $32 \%$. In this phantom each voxel had a label indicating the percentages of fibroglandular and adipose tissue present (100/0, 75/25, 50/50, 25/75, 0/100) or skin. Central slices of the two phantoms are shown in Figure 2 (e) and (f), respectively.

For all the breast models, the compositions of skin, adipose and fibroglandular tissue from Hammerstein et al. ${ }^{45}$ were used. Cooper's ligaments and blood vessels were assumed to have the composition of adult skeletal muscle from ICRU Report $46^{46}$, as previously 
suggested by Ullman et $\mathrm{al}^{47}$. Ducts were simulated using fibroglandular tissue and adipose tissue was used for cavities.

\section{II.C. Simple scatter calculations}

The method estimates the scattered photons distributions for the heterogeneous models (subscript het) using information from prior Monte Carlo simulations generated for the equivalent homogeneous phantom (subscript hom) of lower resolution. After each Monte Carlo simulation experiment using the homogeneous phantom, $P(x, y)$ and $S(x, y)$ are stored and $\operatorname{SPR}(x, y)$ is calculated as described in section 2.1. Then, a $2 \times 2 \mathrm{~cm}^{2}$ ROI located in the detector plane at a distance $\mathrm{R} / 2$ from the chest wall is used to calculate the average scatter $\left(S_{\text {homROI }}\right)$ and SPR $\left(S P R_{\text {homROI }}\right)$ signal. 西

$$
S P R_{\text {homROI }}=\frac{\sum_{R O I} S P R(x, y)}{N_{R O I}},
$$

where $N_{R O I}$ is the number of pixels included within the ROI, i.e. 400 for the $1 \mathrm{~mm}^{2}$ pixels image used in the simulations. This ROI is used as a reference area centred in each projected image. It is used as described below to normalise the SPR and scattered radiation images. In order to keep the ROI centred, avoiding the edges of the breast image, it is shifted laterally for projection angles greater than 0 degrees. This displacement was calculated using the projection of a central point at half height of the breast. Sample ROIs for 0 and 25 degrees projection angles are shown in figure 3(a).

The first stage in the calculation of the scatter for the inhomogeneous phantom is to normalise the homogeneous scatter map to the average scatter within the ROI using $S_{\text {homROI }}$ : 


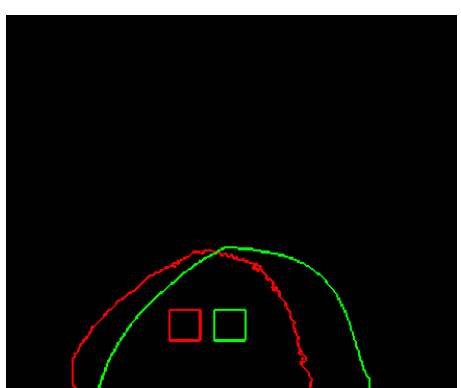

(a)

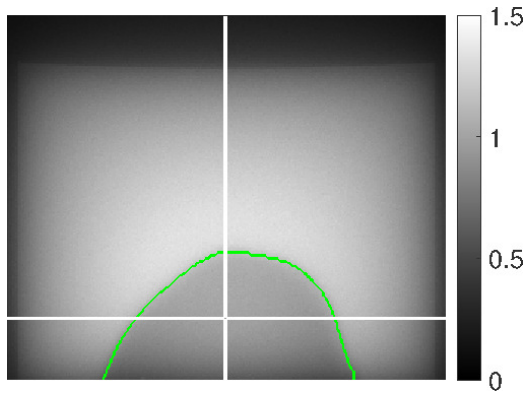

(b)

Figure 3: (a) Projected breast contours and corresponding ROIs for projection angles of 0 and 25 degrees are illustrated in green and red, respectively. (b) Normalised scatter map, $S_{\text {hom }}^{\prime}(x, y)$, for an homogeneous phantom $(\mathrm{T}=6 \mathrm{~cm} ; \mathrm{R}=8 \mathrm{~cm} ; \mathrm{G}=17 \%)$ at $\theta=0$ degrees. The contour of the projected breast is shown in green and the white lines correspond to the location of intensity profiles shown below.

$$
S_{\text {hom }}^{\prime}=\frac{S_{\text {hom }}(x, y)}{S_{\text {homROI }}} .
$$

To estimate the scatter signal in the corresponding heterogeneous model $\left(S_{\text {hetROI }}\right)$, the primary radiation map within the ROI $\left(P_{\text {hetROI }}\right)$ is calculated using a ray tracing algorithm (Siddon $1985^{48}$ ) and then multiplied by the average $S P R$ in the ROI $\left(S P R_{\text {homoROI }}\right)$ :

$$
S_{\text {hetROI }}=S P R_{\text {homROI }} \times P_{\text {hetROI. }}
$$

Finally, the scatter signal across the breast phantom, $S_{h e t}(x, y)$, can be estimated as:

$$
S_{\text {het }}(x, y)=S_{\text {hom }}^{\prime}(x, y) \times S_{\text {hetROI }}
$$

This process was implemented using Matlab R2018a (MathWorks; Natick, Massachusetts, USA) and the 2D scattered radiation distribution was calculated in 15-20 seconds using the pre-calculated Monte Carlo data. A sample distribution of $S_{h o m}^{\prime}(x, y)$ is shown in Figure 3(b). 
In order to evaluate the accuracy of the proposed scatter field estimation, relative error maps $\left(\epsilon_{s}(x, y)\right)$ were calculated between the proposed method $\left(S_{\text {het }}(x, y)\right)$ and direct Monte Carlo simulations using the corresponding heterogeneous phantom $\left(S_{\text {het MC }}(x, y)\right)$, where the latter represents the ground truth:

$$
\epsilon_{S}(x, y)=\frac{S_{\text {het }}(x, y)-S_{\text {hetMC }}(x, y)}{S_{\text {hetMC }}(x, y)} \times 100
$$

A target $\epsilon_{S}$ of $10 \%$ is proposed as an acceptable error. Since SPR values range between 0.2 and 1.4 in DBT geometries for breast thicknesses between 2 to $8 \mathrm{~cm}^{11}$, a $10 \%$ error corresponds to errors between 3 and $5.5 \%$ in the final $\mathrm{P}+\mathrm{S}$ image.

\section{Results}

\section{III.A. Scatter fields comparison of homogeneous and voxelised phantom}

Figure 4 shows sample intensity profiles of $S_{\text {hom }}^{\prime}(x, y)$ for different breast thicknesses , glandularities and projection angles. Note the similar normalised signal intensity between them within most of the projected breast area thanks to the normalisation scheme used, with the largest differences occurring near the edges of the breast model (dashed vertical lines).

Figure 5 shows the value of the relative error $\epsilon_{S}(\mathrm{x}, \mathrm{y})$ of the estimated scatter field for selected breast models of different sizes and glandularities for the 0 degree projection angle. Signed maps are presented and only values within the projected breast area are shown. Such breast areas were automatically segmented using thresholding. The legends represent the relative error in percentage. Overestimation of the scatter method is shown as positive values (green, yellow, orange), while underestimation is shown as negative values (cyan, light and 


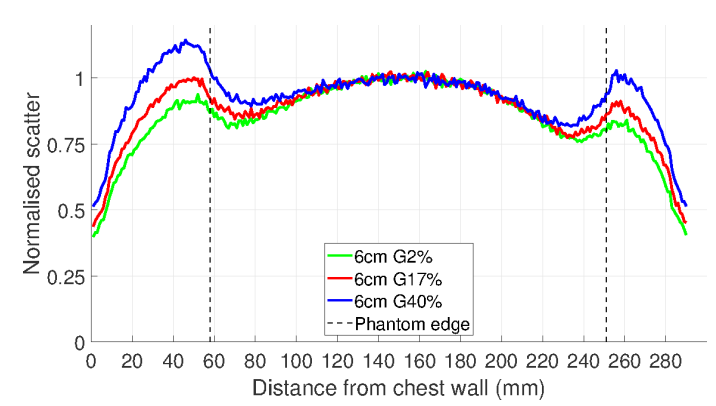

(a)

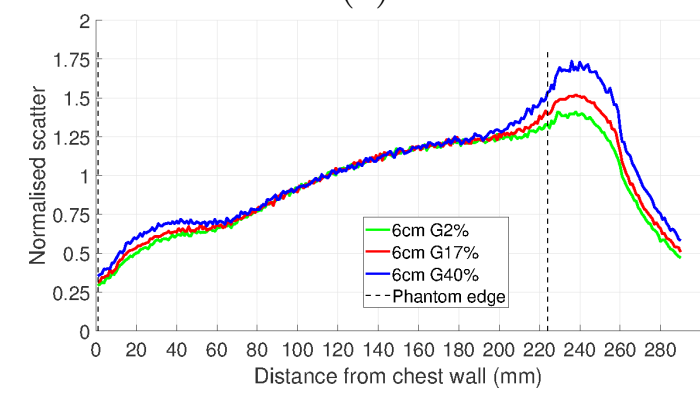

(c)

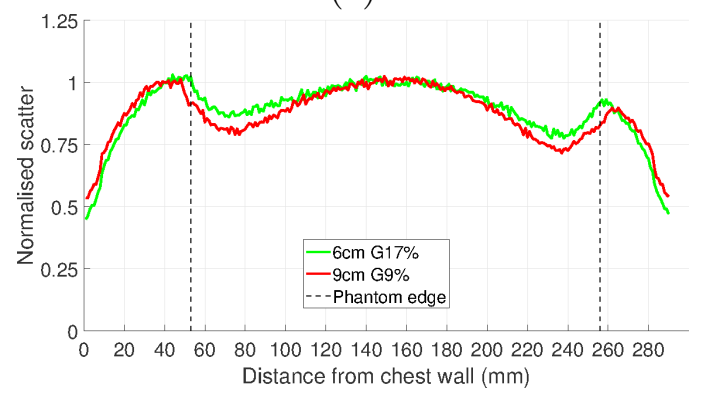

(e)

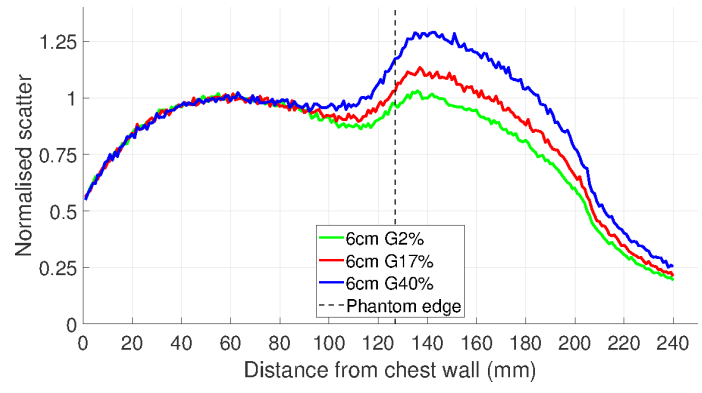

(b)

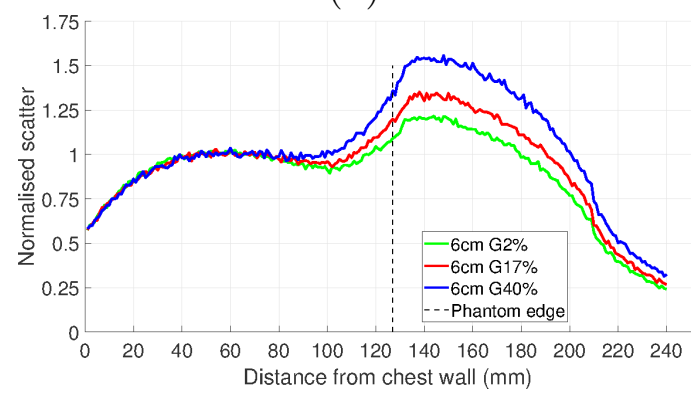

(d)

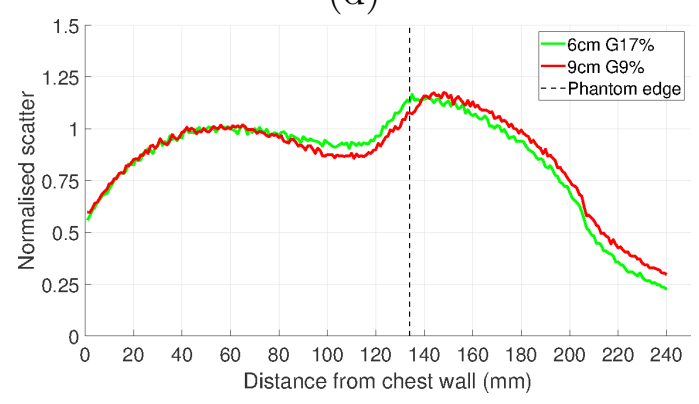

(f)

Figure 4: Horizontal (first column) andvertical (second column) intensity profiles of $S_{\text {hom }}^{\prime}(x, y)$ as illustrated in Figure 3(b). First and second rows correspond to a phantom of $\mathrm{T}=6 \mathrm{~cm}, \mathrm{R}=12 \mathrm{~cm}$ and different glandularities $(2,17$ and $40 \%)$ simulated at $\theta=0$ and $\theta=25$ degrees, respectively. Bottom row (e-f) represents phantoms of $\mathrm{R}=12 \mathrm{~cm}$ and thickness of $6 \mathrm{~cm}(\mathrm{G}=17 \%)$ and $9 \mathrm{~cm}(\mathrm{G}=9 \%)$ for $\theta=0$ degrees. Vertical dashed lines represent the projected edge or boundary of the phantom.

dark blue). A zero error is shown as green. To calculate these differences quantitatively, the average and standard deviation of the modulus is calculated within the projected breast region. The observed modular average and standard deviation differences are $1.6 \pm 1.3 \%$ (minimum $-5.9 \%$, maximum 19.3\%), $2.7 \pm 2.8 \%$ (minimum $-7.9 \%$, maximum $21.6 \%$ ) and 1.7 $\pm 1.3 \%$ (minimum $-9.5 \%$, maximum $21.5 \%$ ) for Figure 5(a), (b) and (c), respectively. For all these three cases, $97 \%$ of the projected breast area shows differences equal to or less than 
$10 \%$, which was set as the maximum difference permitted (detailed information is provided below).

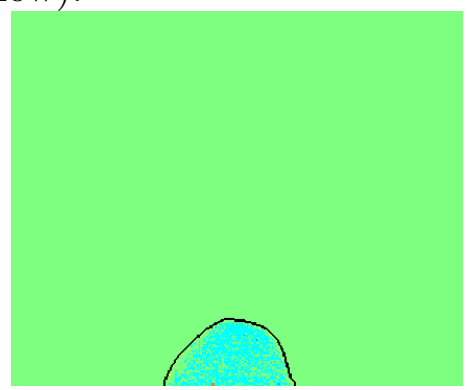

(a)

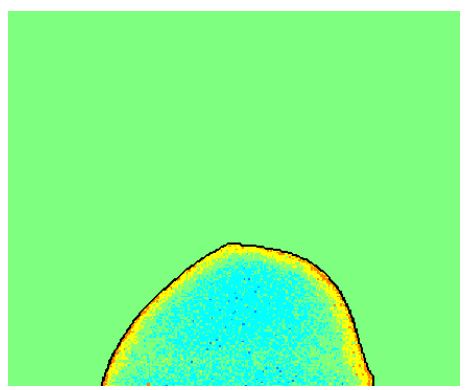

(b)

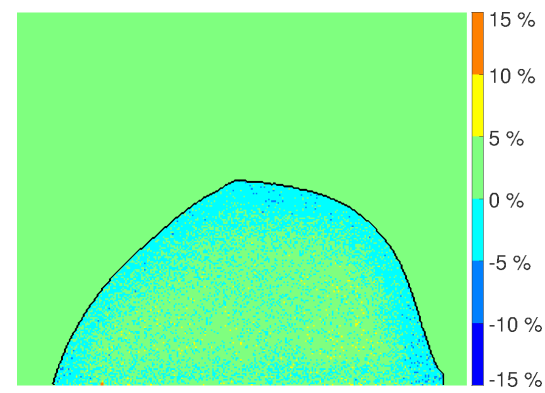

(c)

Figure 5: Relative error map, $\epsilon_{S}(\mathrm{x}, \mathrm{y})$, for several simulated breast models for 0 degree projection angles: (a) $\mathrm{T}=3 \mathrm{~cm}, \mathrm{R}=4 \mathrm{~cm}$ and $\mathrm{G}=35 \%$; (b) $\mathrm{T}=6 \mathrm{~cm}, \mathrm{R}=8 \mathrm{~cm}$ and $\mathrm{G}$ $=17 \%$ and $(\mathrm{c}) \mathrm{T}=9 \mathrm{~cm}, \mathrm{R}=12 \mathrm{~cm}$ and $\mathrm{G}=9 \%$. Breast edge is highlighted with a solid black line.

After fixing $\mathrm{T}(6 \mathrm{~cm}), \mathrm{R}(12 \mathrm{~cm})$ and $\theta$ (0 degree), $\epsilon_{S}(\mathrm{x}, \mathrm{y})$ for different glandularities $(2$, 17 and $40 \%$ ) is shown in figure 6 . The corresponding average and standard deviation moduli of the errors are $2.1 \pm 1.4 \%$ (minimum $-4.6 \%$, maximum 23.3\%), $1.9 \pm 1.4 \%$ (minimum 8.1\%, maximum $25.4 \%$ ) and $3.5 \pm 2.8 \%$ (minimum $-15.2 \%$, maximum $23.6 \%$ ). The fractions of the projected breast with $\epsilon_{S}(\mathrm{x}, \mathrm{y})$ below $10 \%$ are $100 \%, 100 \%$ and $97 \%$ respectively. The values of $\epsilon_{S}(\mathrm{x}, \mathrm{y})$ observed within the projected breast area are below $5 \%$ (cyan and green) in the central area of the breast.

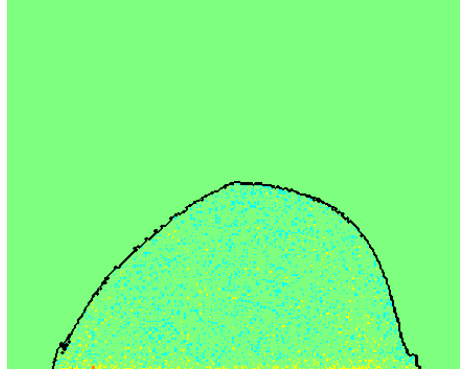

(a)

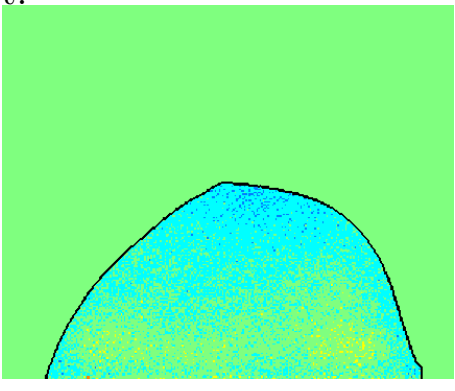

(b)

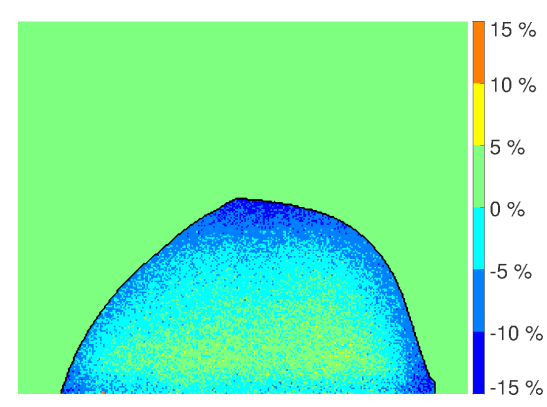

(c)

Figure 6: Relative error maps $\epsilon_{S}(\mathrm{x}, \mathrm{y})$ for a phantom with $\mathrm{T}=6 \mathrm{~cm}, \mathrm{R}=12 \mathrm{~cm}$ and $\theta=0$ degrees. Glandularities correspond to (a) 2, (b) 17 and (c) 40\%. Breast edge is highlighted with a solid black line.

252 Figure 7 depicts, for a given phantom $(\mathrm{T}=6 \mathrm{~cm}, \mathrm{R}=8 \mathrm{~cm}, \mathrm{G}=17 \%)$, the variations 
of $\epsilon_{S}(\mathrm{x}, \mathrm{y})$ with projection angle $(0,7.5$ and 25 degrees $)$. As expected, the projected breast is shifted towards the left edge of the image as the x-ray source is moved towards the right side, as does the ROI as presented in Figure 3(b). Average and standard deviation modulus values within projected breast area are $2.7 \pm 2.8 \%$ (minimum $-7.9 \%$, maximum $21.6 \%$ ), 2.8 $\pm 2.7 \%$ (minimum $-6.2 \%$, maximum $21.2 \%$ ) and $2.9 \pm 2.6 \%$ (minimum $-8.0 \%$, maximum $25.5 \%$ ) for $0,7.5$ and 25 degrees, respectively. For these cases, $99 \%$ of the breast area has differences from the ground truth data equal to or below $10 \%$.

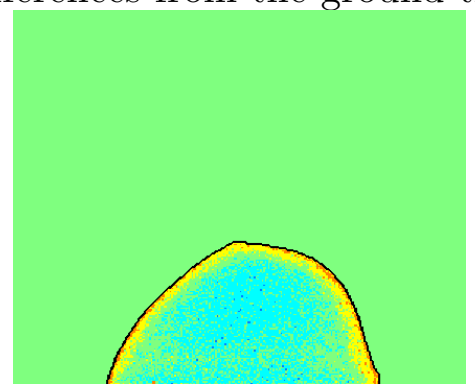

(a)

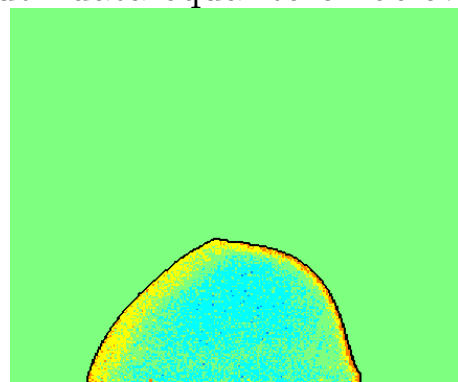

(b)

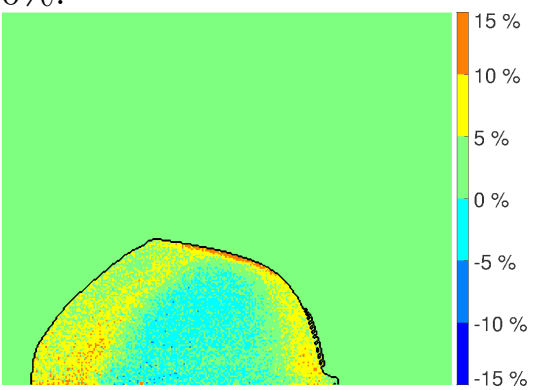

(c)

Figure 7: Relative error maps $\epsilon_{S}(\mathrm{x}, \mathrm{y})$ for a $6 \mathrm{~cm}$ thick and $\mathrm{R}=8 \mathrm{~cm}$ breast phantom $(\mathrm{G}=17 \%$ ) at (a) 0, (b) 7.5 and (c) 25 degrees' projection angles. Breast edge is highlighted with a solid black line.

\section{III.B. Scattered radiation for different glandularity patterns}

As previously described, for the phantom $(\mathrm{T}=6 \mathrm{~cm}, \mathrm{R}=12 \mathrm{~cm}, \mathrm{G}=17 \%)$, three different fibroglandular distribution patterns were produced (Figure 2). Figure 8 shows $\epsilon_{S}(\mathrm{x}, \mathrm{y})$ for this phantom, and a 0 degrees projection. The mean and standard deviation modulus errors observed within the projected breast area are $3.4 \pm 2.6 \%$ (minimum $-13.3 \%$, maximum $17.0 \%$ ), $2.6 \pm 1.8 \%$ (minimum $-11.2 \%$, maximum $27.7 \%$ ) and $2.4 \pm 1.9 \%$ (minimum $-11.6 \%$, maximum 22.3\%), for each of the cases shown. 99\% of the projected breast areas have $\epsilon_{S}(\mathrm{x}, \mathrm{y})$ below $10 \%$. 


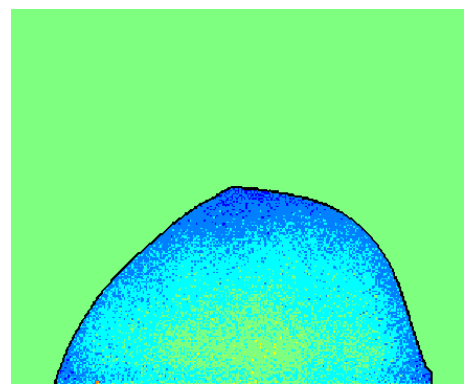

(a)

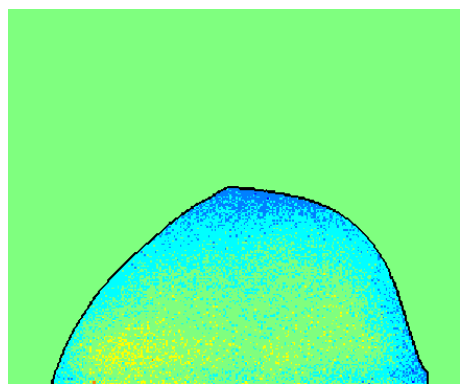

(b)

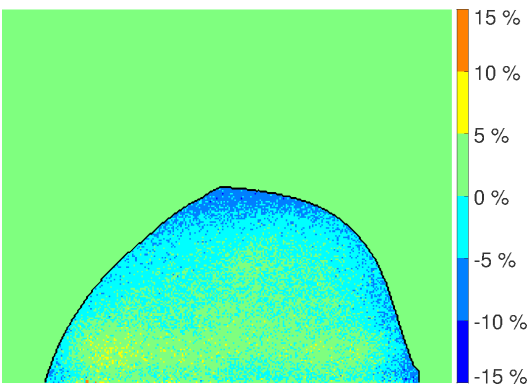

(c)

Figure 8: Relative error maps $\epsilon_{S}(\mathrm{x}, \mathrm{y})$ for a $6 \mathrm{~cm}$ thick and $12 \mathrm{~cm}$ radius phantom $(\mathrm{G}=17 \%$, $\theta=0$ degrees), and different fibroglandular pattern distributions (P1, P2, P3). Breast edge is highlighted with a solid black line.

\section{III.C. Scattered radiation using other breast models}

Differences between the estimated and ground truth scattered radiation, i.e. $\epsilon_{S}(\mathrm{x}, \mathrm{y})$, are shown in Figure 9 for the $5 \mathrm{~cm}$ thick compressed UPenn breast model $(\mathrm{G}=20 \%)$ and the 6 cm thick compressed Duke University breast phantom $(\mathrm{G}=32 \%)$. The average and standard deviation of the modulus error are $1.7 \pm 1.3 \%$ (minimum $-7.8 \%$, maximum $8.0 \%$ ) and $5.1 \pm$ $2.9 \%$ (minimum -15.3\%, maximum $7.4 \%$ ) for the UPenn and Duke phantoms, respectively , at $\theta=0$ degrees. $97 \%$ of area of the UPenn projected images show differences below $5 \%$ for all simulated projection angles, whereas $93 \%$ of the areas of Duke phantom projected images show differences up to $10 \%$.

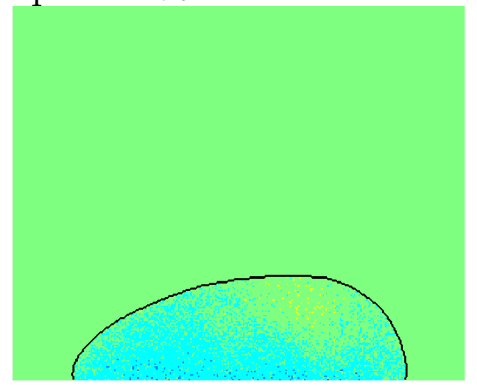

(a)

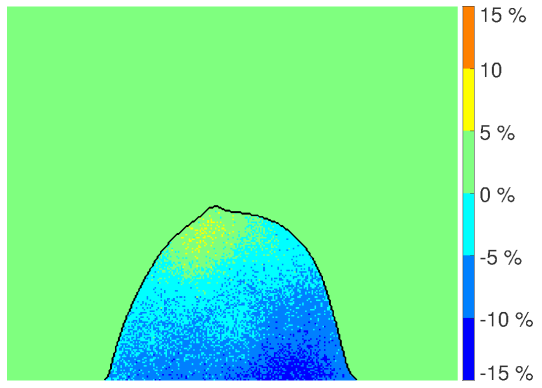

(b)

Figure 9: $\epsilon_{S}(\mathrm{x}, \mathrm{y})$ using (a) $5 \mathrm{~cm}$ thick UPenn breast model $(\mathrm{G}=20 \%)$ and (b) $6 \mathrm{~cm}$ thick Duke University breast model $(\mathrm{G}=32 \%)$, acquired at $\theta=0$ degrees. Breast edge is highlighted with a solid black line. 
278

279

280 errors $\left(\epsilon_{S} \leq 2 \%, 5 \% 10 \%, 12 \%\right.$ and $\left.15 \%\right)$ are given.

\section{Table 2: Error analysis for each phantom and acquired} geometry.

\begin{tabular}{|c|c|c|c|c|c|c|c|c|c|c|c|c|c|}
\hline Phantom & $\begin{array}{c}\mathrm{T} \\
(\mathrm{cm})\end{array}$ & $\begin{array}{c}\mathrm{R} \\
(\mathrm{cm})\end{array}$ & $\begin{array}{c}\mathrm{G} \\
(\%)\end{array}$ & $\begin{array}{c}\theta \\
\text { (degrees) }\end{array}$ & $\begin{array}{c}\text { Mean } \epsilon_{S} \\
(\%)\end{array}$ & $\begin{array}{c}\text { Stdev. } \epsilon_{S} \\
(\%)\end{array}$ & $\begin{array}{c}\operatorname{Min} . \epsilon_{S} \\
(\%)\end{array}$ & $\begin{array}{c}\operatorname{Max} . \epsilon_{S} \\
(\%)\end{array}$ & $\begin{array}{c}\epsilon_{S} \leq 2 \% \\
(\%)\end{array}$ & $\begin{array}{c}\epsilon_{S} \leq 5 \% \\
(\%)\end{array}$ & $\epsilon_{S} \leq 10 \%$ & $\epsilon_{S} \leq 12 \%$ & $\epsilon_{S} \leq 15 \%$ \\
\hline \multirow{33}{*}{ Elangovan et al ${ }^{33}$} & \multirow{6}{*}{3} & \multirow{3}{*}{4} & \multirow{3}{*}{35} & 0 & 1.6 & 1.3 & -5.9 & 19.3 & 68.8 & 98.3 & 99.9 & 99.9 & 100.0 \\
\hline & & & & 7.5 & 1.6 & 1.3 & -5.8 & 18.3 & 70.7 & 98.0 & 99.8 & 99.9 & 99.9 \\
\hline & & & & 25 & 1.7 & 1.4 & -5.8 & 20.8 & 65.9 & 97.9 & 99.9 & 99.9 & 100.0 \\
\hline & & \multirow{3}{*}{8} & \multirow{3}{*}{35} & 0 & 2.1 & 1.7 & -7.4 & 13.1 & 57.5 & 93.8 & 99.9 & 100.0 & 100.0 \\
\hline & & & & 7.5 & 2.0 & 1.7 & -7.8 & 16.2 & 58.6 & 93.7 & 99.8 & 100.0 & 100.0 \\
\hline & & & & 25 & 2.2 & 1.9 & -7.7 & 16.3 & 58.3 & 90.9 & 99.8 & 100.0 & 100.0 \\
\hline & \multirow{21}{*}{6} & \multirow{3}{*}{8} & \multirow{3}{*}{17} & 0 & 2.7 & 2.8 & -7.9 & 21.6 & 54.2 & 84.1 & 96.6 & 98.9 & 99.8 \\
\hline & & & & 7.5 & 2.8 & 2.7 & -6.2 & 21.2 & 52.5 & 83.2 & 97.1 & 98.8 & 99.8 \\
\hline & & & & 25 & 2.9 & 2.6 & -8.0 & 25.5 & 47.2 & 80.5 & 98.1 & 99.3 & 99.9 \\
\hline & & \multirow{18}{*}{12} & \multirow{3}{*}{2} & 0 & 2.1 & 1.4 & -4.6 & 23.3 & 52.9 & 96.5 & 100.0 & 100.0 & 100.0 \\
\hline & & & & 7.5 & 1.9 & 1.4 & -4.2 & 26.1 & 58.2 & 97.2 & 100.0 & 100.0 & 100.0 \\
\hline & & & & 25 & 1.8 & 1.3 & -7.3 & 11.8 & 63.7 & 97.6 & 100.0 & 100.0 & 100.0 \\
\hline & & & \multirow{3}{*}{17} & 0 & 1.9 & 1.4 & -8.1 & 25.4 & 60.4 & 97.1 & 100.0 & 100.0 & 100.0 \\
\hline & & & & 7.5 & 1.9 & 1.4 & -9.7 & 23.0 & 61.1 & 97.2 & 100.0 & 100.0 & 100.0 \\
\hline & & & & 25 & 1.9 & 1.5 & -9.0 & 10.3 & 59.3 & 96.1 & 100.0 & 100.0 & 100.0 \\
\hline & & & \multirow{3}{*}{$17(\mathrm{P} 1)$} & 0 & 3.4 & 2.6 & -13.3 & 17.0 & 37.4 & 73.6 & 98.5 & 99.9 & 100.0 \\
\hline & & & & 7.5 & 3.6 & 2.7 & -14.0 & 16.5 & 34.6 & 70.7 & 98.2 & 99.9 & 100.0 \\
\hline & & & & 25 & 4.6 & 3.0 & -17.0 & 4.8 & 22.5 & 59.8 & 94.9 & 98.8 & 100.0 \\
\hline & & & \multirow{3}{*}{17 (P2) } & 0 & 2.6 & 1.8 & -11.2 & 27.7 & 45.0 & 89.1 & 100.0 & 100.0 & 100.0 \\
\hline & & & & 7.5 & 2.4 & 1.8 & -11.2 & 24.1 & 48.7 & 89.8 & 99.9 & 100.0 & 100.0 \\
\hline & & & & 25 & 2.8 & 2.2 & -13.6 & 10.5 & 45.0 & 83.7 & 99.3 & 100.0 & 100.0 \\
\hline & & & \multirow{3}{*}{17 (P3) } & 0 & 2.4 & 1.9 & -11.6 & 22.3 & 51.6 & 89.7 & 99.9 & 100.0 & 100.0 \\
\hline & & & & 7.5 & 2.4 & 1.9 & -10.9 & 23.4 & 52.3 & 89.3 & 99.9 & 100.0 & 100.0 \\
\hline & & & & 25 & 2.8 & 2.1 & -13.3 & 10.2 & 44.4 & 85.2 & 99.6 & 100.0 & 100.0 \\
\hline & & & \multirow{3}{*}{40} & 0 & 3.5 & 2.8 & -15.2 & 23.6 & 38.7 & 73.6 & 96.8 & 99.4 & 100.0 \\
\hline & & & & 7.5 & 3.2 & 2.5 & -14.2 & 22.8 & 40.8 & 77.9 & 98.6 & 99.8 & 100.0 \\
\hline & & & & 25 & 5.2 & 3.2 & -16.5 & 5.5 & 16.2 & 53.0 & 91.5 & 96.6 & 99.8 \\
\hline & \multirow{6}{*}{9} & \multirow{3}{*}{12} & \multirow{3}{*}{9} & 0 & 1.7 & 1.3 & -9.5 & 21.5 & 63.4 & 98.4 & 100.0 & 100.0 & 100.0 \\
\hline & & & & 7.5 & 1.7 & 1.3 & -7.5 & 24.6 & 64.9 & 98.5 & 100.0 & 100.0 & 100.0 \\
\hline & & & & 25 & 1.7 & 1.3 & -9.0 & 12.1 & 65.5 & 97.9 & 100.0 & 100.0 & 100.0 \\
\hline & & \multirow{3}{*}{16} & \multirow{3}{*}{9} & 0 & 1.7 & 1.3 & -7.0 & 10.4 & 65.3 & 98.2 & 100.0 & 100.0 & 100.0 \\
\hline & & & & 7.5 & 1.5 & 1.2 & -11.0 & 9.8 & 70.3 & 98.7 & 100.0 & 100.0 & 100.0 \\
\hline & & & & 25 & 1.6 & 1.2 & -10.0 & 10.7 & 68.2 & 98.4 & 100.0 & 100.0 & 100.0 \\
\hline \multirow{3}{*}{ Bakic et al ${ }^{34}$} & & & & 0 & 1.7 & 1.3 & -7.8 & 8.0 & 63.1 & 98.4 & 100.0 & 100.0 & 100.0 \\
\hline & 5 & 5 & 20 & 7.5 & 1.7 & 1.3 & -7.4 & 6.6 & 64.8 & 98.2 & 100.0 & 100.0 & 100.0 \\
\hline & & & & 25 & 1.8 & 1.4 & -8.7 & 7.3 & 61.3 & 97.2 & 100.0 & 100.0 & 100.0 \\
\hline & & & & 0 & 5.1 & 2.9 & -15.3 & 7.4 & 16.0 & 52.0 & 94.1 & 98.7 & 100.0 \\
\hline Li et $\mathrm{al}^{35}$ & 6 & 9 & 32 & 7.5 & 5.2 & 3.0 & -16.2 & 7.4 & 15.5 & 50.2 & 92.6 & 98.3 & 100.0 \\
\hline & & & & 25 & 5.0 & 3.1 & -15.2 & 6.5 & 20.0 & 54.0 & 93.0 & 98.7 & 100.0 \\
\hline
\end{tabular}

\section{${ }_{281}$ IV. Discussion}

As observed in Figure 4, the use of the ROI produces a normalised scatter signal of approximately 1 across the central region of the projected breast. As expected, this quantity increases towards the edges of the breast due to scattered radiation from the compression 
paddle and breast support. This effect is also observed when the projection angle is increased, even though the scatter signal distribution is no longer symmetric (Figure 4(c)). Scattered radiation originating in parts of the DBT system (e.g. compression paddle and breast support table) is considered in this work. However, the photons scattered from the patient's body reaching the detector are not taken into account. Although several works having simulated a patient 's body for scatter estimation ${ }^{11,40}$, the magnitude of this scatter contribution was not discussed. We believe that the majority of scattered photons from the body will be mainly absorbed by the breast and little signal will reach the image receptor. They will probably represent a small fraction of the total scattered radiation, compared to that from the breast and compression paddle, however further investigation is needed to confirm this.

Through Figures 5 to 8, we have demonstrated that the proposed work can accurately estimate scatter fields for different T, G and $\theta$, showing average modular differences below $5.2 \%$ for all simulated breast models (Table 2). Figure 7 shows how the errors in the left side of the periphery of the breast (yellow region) increase as the x-ray source is moved towards the right side of the figure. As described in the literature ${ }^{12}$, this overestimation in scattered radiation is due to the increase of the airgap distance traversed by the x-ray photons between the bottom surface of the breast and the breast support, which is not properly modelled. Likewise, the UPenn and Duke breast phantoms (Figures 9) have shown average errors below $5.2 \%$. The errors are mostly within $5 \%$ (green or cyan) in the central area, where the ROI is selected. Therefore, this supports the ascertion that scattered radiation from homogeneous breast models can be used to approximate scatter field produced from heterogeneous models ${ }^{32}$. The largest discrepancies were found for the highest density $(\mathrm{G}$ $=40 \%$ ), as observed in figure 6 . However, this level of glandularity is rarely seen in routine breast screening, but may be pertinent in cases where younger women with a known history of breast disease are subject to mammography. This particular breast model shows larger differences (10-15\%) near the edges of the breast, where there is more scattered radiation 
from the compression paddle. To compute the scatter signal, the proposed method takes the SPR reference value in the center of the homogeneous phantom and this will differ largely from the SPR value of the peripheral area, as previously noticed in the literature where differences up to $30 \%$ in SPR were observed for DBT geometries ${ }^{11}$. These low differences will be more relevant for larger breast densities. However, if using a VCT based on an M alternative forced choice (M-AFC) experiments, image patches from areas close to the edge of the breast might not be important to assess the imaging performance of a system. In addition, the amount of scatter from the compression paddle depends on its thickness and composition, and will thus differ between manufacturers.

Since the scatter field depends on the breast shape and composition, new phantoms will require running new Monte Carlo simulations. The use of low-resolution phantoms can reduce the computational time more than $40 \%$ with respect to that for high-resolution phantoms, while maintaining accuracy. However, given a fixed breast phantom size and glandularity, the same pre-calculated Monte Carlo results can be used for a wide range of distributions of fibroglandular tissue without the need for repeat simulations (Figure 8). It has been demonstrated that this scatter field estimation method works for three different types of breast models. The breast models used in this study have a compressed shape which varies slowly for the different configurations, thus new combinations could be obtained by interpolating the pre-calculated data for selected $\mathrm{T}, \mathrm{G}$ and $\theta$.

Note that, as commented earlier, only errors within the breast area were computed because the background does not have clinical relevance. Since automated segmentation was used to define the outline of the projected breast, slightly unrealistic breast edges might have been used to estimate $\epsilon_{S}(\mathrm{x}, \mathrm{y})$ for the largest projected breasts (Figure $7(\mathrm{c})$ ).

For all the phantoms simulated, the average SPR values within the ROIs range between 0.3 and 1.4. Therefore our target $10 \%$ error in scatter signal represents an error in the total simulated image $(P+S)$ below $6 \%$. We have demonstrated that our method estimates 
scattered radiation with a $5 \%$ error within $87 \%$ of the projected breast area, on average. Therefore our approach for VCT schemes allows the rapid estimation of scatter fields in less than 20 seconds with errors of $3 \%$ within most of the area of the synthetic $(P+S)$ image. By contrast we note that in certain VCT schemes scattered radiation is sometimes ignored $^{49,50}$.

Similarly, a recent VCT scheme ${ }^{24}$ has shown fast generation of synthetic breast images, including scattered radiation. The average time to generate images for one patient was 8 central processing unit hours and 0.5 graphic processing unit hours, thus requiring good computer resources. By contrast, our approach could be run using a simple laptop since pre-calculated Monte Carlo simulations are used.

In addition, our method has potential to be applied in clinical DBT, although since it relies on the geometry of mathematical breast models, its accuracy as a scatter field reduction tool needs to be further tested.

\section{Conclusions}

A fast and simple method has been developed to estimate the $2 \mathrm{D}$ distribution of scattered radiation in VCT schemes which simulate DBT. A considerable reduction in the computational time has been shown with respect to other approaches ${ }^{12,27}$. The proposed method, which uses pre-calculated Monte Carlo data using low-resolution breast models, takes less than 20 seconds to estimate the scatter field across the projected breast.

For the range of breast phantoms used in this study, the differences between the proposed scatter field estimation method and the ground truth data (direct Monte Carlo with high resolution anthropomorphic phantoms) are on average below 5.2\% (minimum -17.0\%, maximum $27.7 \%$ ) within the projected breast area. It was found that, on average, the scatter field estimation method can estimate scattered radiation with an error of $10 \%$ (or less) in 
$99 \%$ of the projected phantom area. If the accuracy requirement is set to $5 \%$ differences in scatter signal, $87 \%$ of all analysed cases are below this threshold. Thus, the method, which has been validated using three of the most commonly used breast models in the field, is a useful tool to accurately insert scattered radiation into VCT schemes.

\section{Acknowledgments}

This work is part of the OPTIMAM2 project funded by Cancer Research UK (grant number: C30682/A17321).

\section{Conflict of Interest}

The authors have no relevant conflicts of interest to disclose. 


\section{अ7 References}

373

1 Barnes, GT. Contrast and scatter in x-ray imaging. Radiographics. 1991;11(2):307-323. doi: 10.1148/radiographics.11.2.2028065.

2 Ducote JL, Molloi S. Scatter correction in digital mammography based on image deconvolution. Physics in Medicine and Biology. 2010; 55(5):1295-1309. doi: 10.1088/0031$9155 / 55 / 5 / 003$.

3 Chan HP, Lam KL, Wu Y. Studies of performance of antiscatter grids in digital radiography: effects on signal-to-noise ratio. Medical Physics. 1990;17(4):655-664. doi: $10.1118 / 1.596496$.

4 Neitzel U. Grids or airgaps for scatter reduction in digital radiography: a model calculation. Medical Physics. 1992;19(2):475-481. doi: 10.1118/1.596836.

5 Veldkamp WJH, Thijssen, MAO, Karssemeijer N. The value of scatter removal by a grid in full field digital mammography. Medical Physics. 2003;30(7):1712-1718. doi: $10.1118 / 1.1584044$.

6 Wu G, Mainprize JG, Boone JM, Yaffe MJ. Evaluation of scatter effects on image quality for breast tomosynthesis. Medical Physics. 2009;36(10):4425-4432. doi: $10.1118 / 1.3215926$.

7 Dance DR, Day GJ. The computation of scatter in mammography by Monte Carlo methods. Physics in Medicine and Biology. 1984;29(3):237-247. doi: 10.1088/0031$9155 / 29 / 3 / 003$

8 Highnam RP, Brady JM, Shepstone BJ. Computing the Scatter Component of Mammographic Images. IEEE Transactions on Medical Imaging. 1994;13(2):301-313. doi: 10.1109/42.293922. 
9 Boone JM, Cooper VN. Scatter/primary in mammography: Monte Carlo validation. Medical Physics. 2000;27(8):1818-1831. doi: 10.1118/1.1287052.

10 Nykanen K, Siltanen S. X-ray scattering in full-field digital mammography. Medical Physics. 2003; 30(7):1864-1873. doi: 10.1118/1.1584160.

11 Sechopoulos I, Suryanarayanan S, Vedantham S, D'Orsi C, Karellas A. Scatter radiation in digital tomosynthesis of the breast. Medical Physics. 2007;34(2):564-576. doi: $10.1118 / 1.2428404$.

12 Diaz O, Dance DR, Young KC, Elangovan P, Bakic PR, Wells K. Estimation of scattered radiation in digital breast tomosynthesis. Physics in Medicine and Biology. 2014; 59(15):4375-4390. doi: 10.1088/0031-9155/59/15/4375.

$13 \mathrm{Wu}$ G, Inscoe CR, Calliste J, et al. Estimating scatter from sparsely measured primary signal. Journal of Medical Imaging. 2017;4(1):013508. doi: 10.1117/1.JMI.4.1.013508.

14 Feng SS, Sechopoulos I. A software-based x-ray scatter correction method for breast tomosynthesis. Medical Physics. 2011;38(12):6643-6653. doi: 10.1118/1.3659703.

15 Feng SS, D’Orsi CJ, Newell MS, Seidel RL, Patel B, Sechopoulos I. X-ray scatter correction in breast tomosynthesis with a precomputed scatter map library. Medical Physics. 2014;41(3):031912. doi: 10.1118/1.4866229.

16 Wang, A, Shapiro E, Yoon S, et al. Asymmetric Scatter Kernels for Software-Based Scatter Correction of Gridless Mammography. In:Proc. SPIE 9412, Medical Imaging 2015: Physics of Medical Imaging, 94121I. doi: 10.1117/12.2081501

17 Green FH, Veale MC, Wilson MD, Seller P, Scuffham J, Pani S. Scatter free imaging for the improvement of breast cancer detection in mammography. Physics in Medicine and Biology. 2006;61(20):7246. doi: 10.1088/0031-9155/61/20/7246. 
18 Marimon E, Nait-Charif H, Khan A, Marsden PA, Diaz O. Detailed analysis of scatter contribution from different simulated geometries in X-ray detectors. In: Breast Imaging. IWDM 2016. Lecture Notes in Computer Science. 2016;9699:203-210. doi: 10.1007/9783-319-41546-8_27.

19 Kim K, Lee T, Seong Y, et al. Fully iterative scatter corrected digital breast tomosynthesis using GPU-based fast Monte Carlo simulation and composition ratio update. Medical Physics. 2015;42(9):5342-5355. doi: 10.1118/1.4928139

20 Gong X, Glick SJ, Liu B, Vedula, AA, Thacker S. A computer simulation study comparing lesion detection accuracy with digital mammography, breast tomosynthesis, and cone-beam CT breast imaging. Medical Physics. 2006;33(4):1041-1052. doi: $10.1118 / 1.2174127$

21 Young S, Bakic PR, Myers KJ, Jennings RJ, Park S. A virtual trial framework for quantifying the detectability of masses in breast tomosynthesis projection data. Medical Physics. 2013;40(5): 051914. doi: 10.1118/1.4800501.

22 Elangovan P, Warren L, Mackenzie A, et al. Development and validation of a modelling framework for simulating 2D-mammography and breast tomosynthesis images. Physics in Medicine and Biology. 2014;59(15):4275-4293 . doi: 10.1088/0031-9155/59/15/4275

23 Elangovan P, Mackenzie A, Dance DR, Young KC, Wells K. Lesion detectability in 2Dmammography and digital breast tomosynthesis using different targets and observers. Physics in Medicine and Biology. 2018;63(9):095014. doi: 10.1088/1361-6560/aabd53.

24 Badano A, Graff CG, Badal A, et al. Evaluation of Digital Breast Tomosynthesis as Replacement of Full-Field Digital Mammography Using an In Silico Imaging Trial. JAMA Network Open. 2018;1(7):e185474. doi:10.1001/jamanetworkopen.2018.5474 
25 Elangovan P, Mackenzie A, Dance DR, Young KC, Wells K. Using non-specialist observers in 4AFC human observer studies. In: Proc. SPIE 10132, Medical Imaging 201\%: Physics of Medical Imaging, 1013256 (9 March 2017); doi: 10.1117/12.2255560

26

Kazemi S, Diaz O, Elangovan P, Wells K, Lohstroh A. Comparison of Breast ConeBeam Computed Tomography, 2D Mammography and Digital Breast Tomosynthesis Imaging Modalities, using 4-Alternative Forced Choice Human Observation Study. In: Proc. SPIE 10573, Medical Imaging 2018: Physics of Medical Imaging: 105735I. doi: $10.1117 / 12.2293201$ contrast of discs in synthetic CDMAM images. In:Digital Mammography. IWDM 2010. Lecture Notes in Computer Science. 2010; 6136: 628-663. doi: 10.1007/978-3-642-13666$5 \_85$.

28 Dance DR, Persliden J, Alm Carlsson G. Calculation of dose and contrast for two mammographic grids. Physics in Medicine and Biology. 1992; 37(1): 235-248. doi: $10.1088 / 0031-9155 / 37 / 1 / 017$

Cunha DM, Tomal A, Poletti ME. Evaluation of scatter-to-primary ratio, grid performance and normalized average glandular dose in mammography by Monte Carlo simulation including interference and energy broadening effects. Physics in Medicine and Biology. 2010;55(15):4335-4359. doi: 10.1088/0031-9155/55/15/010.

30 Diaz O, Dance DR, Young KC, Elangovan P, Bakic PR, Wells K. A fast scatter field estimator for digital breast tomosynthesis. In: Proc. SPIE 8313, Medical Imaging 2012: Physics of Medical Imaging, 831305. doi: 10.1117/12.911494.

31 Diaz O, Garcia E, Oliver A, Marti J, Marti R. Scattered radiation in DBT geometries with fexible breast compression paddles: A Monte Carlo simulation study. In: 
Proc. SPIE 10132, Medical Imaging 201\%: Physics of Medical Imaging, 101324G. doi: $10.1117 / 12.2255722$ to estimate scattered radiation in breast tomosythesis?. In: Proc. SPIE 10948, Medical Imaging 2019: Physics of Medical Imaging, 109485J. doi: 10.1117/12.2512287. models for use in multiple alternative forced choice virtual clinical trials. Physics in Medicine and Biology. 2017;62(7):2278-2794. doi: 10.1088/1361-6560/aa622c morphic breast software phantom based upon region-growing algorithm. Medical Physics. 2011; 38(6):3165-3176. doi: 10.1118/1.3590357 3D computerized breast phantom from empirical data. Medical Physics. 2009;36(7):31223131. doi: $10.1118 / 1.3140588$ of montE CarlO RaDiation transport Studies: Report of the APPM Research Committee Task Group 268. Medical Physics. 2018;45(1):e1-e5. doi: 10.1002/mp.12702. Medical Physics. 2013;40(1):014301. doi: 10.1118/1.4770279.

38 Agostinelli S, Allison J, Amako K, et al. Geant4 - a simulation toolkit. Nuclear Instruments and Methods in Physics Research. 2003;506(3):250-303. doi: 10.1016/S01689002(03)01368-8.

39 Allison J, Amako K, Apostolakis J, et al. Geant4 development and applications. IEEE Transactions on Nuclear Science. 2006; 53(1):270-278. doi: 10.1109/TNS.2006.869826. 
40 Sechopoulos I, Ali ES, Badal A, et al. Monte Carlo reference data sets for imaging research: Executive summary of the report of AAPM Research Committee Task Group 195. Medical Physics. 2015;42(10):5679-5691. doi: 10.1118/1.4928676.

41 Marimon E, Nait-Charif H, Khan A, Marsden PA, Diaz O. Scatter reduction for gridless mammography using the convolution-based image post processing technique. In: Proc. SPIE 10132, Medical Imaging 201\%: Physics of Medical Imaging, 101324D. doi: $10.1117 / 12.2255558$.

Boone J, Fewell T, Jennings J. Molybdenum, rhodium and tungsten anode spectral models using interpolating polynomials with applications to mammography. Medical Physics. 1997;24(12):1863-1874. doi: 10.1118/1.598100.

Strudley CJ, Looney P, Young KC. Technical evaluation of Hologic Selenia Dimensions digital breast tomosynthesis system. NHSBSP Equipment Report. 2014; 1307 (v2).

44 Mackenzie A, Thomson EL, Elangovan P, et al. An observer study to assess the detection of calcification clusters using 2D mammography, digital breast tomosynthesis, and synthetic 2D imaging. In: Proc. SPIE 10952, Medical Imaging 2019: Image Perception, Observer Performance, and Technology Assessment, 109520U. doi: 10.1117/12.2506895.

45 Hammerstein GR, Miller DW, White DR, Masterson ME, Woodard HQ, Laughlin, JS. Absorbed radiation dose in mammography. Radiology. 1979; 130:485-491. doi: $10.1148 / 130.2 .485$

46 White DR, Griffith RV, Wilson IJ. Photon, electron, proton and neutron interaction data for body tissues. Report 46, Journal of the International Commission on Radiation Units and Measurements. 1992;24(1). doi: 10.1093/jicru/os24.1.Report46

7 Ullman G, Sandborg M, Hunt R, Dance DR, Alm Carlsson G. Implementation of patholo- 
gies in the Monte Carlo model in chest and breast imaging. Technical Report. Institutionen för radiologi, Universitetet i Linköping. 2003; 94.

48 Siddon RL. Fast calculation of the exact radiological path for the three-dimensional CT array. Medical Physics. 1985; 12(2):252-255. doi: 10.1118/1.595715.

49 Bakic PR, Barufaldi B, Higginbotham D, Weinstein SP, Avanaki AN, et al. Virtual clinical trial of lesion detection in digital mammography and digital breast tomosynthesis. In: Proc. SPIE 10573, Medical Imaging 2018: Physics of Medical Imaging, 1057306. doi: $10.1117 / 12.2294934$.

50 Barufaldi B, Higginbotham D, Bakic PR, Maidment ADA. OpenVCT: a GPUaccelerated virtual clinical trial pipeline for mammography and digital breast tomosynthesis. In: Proc. SPIE 10573, Medical Imaging 2018: Physics of Medical Imaging, 1057358. doi: $10.1117 / 12.2294935$. 


\section{${ }_{524}$ List of Figures}

Fig. 1: Schematic diagram of the geometry used in the Monte Carlo simulations in (a) sagittal and (b) coronal view. The detector tilts with projection angles. Note that the figures are not in scale.

Fig. 2: (a-c) Central slices of $6 \mathrm{~cm}$ thick compressed voxelised breast phantoms, $12 \mathrm{~cm}$ radius and volumetric glandularity 17\% using different patterns (P1-P3). The corresponding homogeneous breast model is shown in $(\mathrm{d})$. Note that the homogeneous breast has a lower resolution. Sample slices of breast models developed at UPenn ${ }^{34}$ and Duke University $^{35}$ are depicted in (e) and (d), respectively. The different tissues has been indicated: Skin,fibroglandular tissue (FT), adipose tissue (AT), Cooper's ligaments (CL), blood vessels (BL) and adipose cavities (AC).

Fig. 3: (a) Projected breast contours and corresponding ROIs for projection angles of 0 and 25 degrees are illustrated in green and red, respectively. (b) Normalised scatter map, $S_{\text {hom }}^{\prime}(x, y)$, for an homogeneous phantom $(\mathrm{T}=6 \mathrm{~cm} ; \mathrm{R}=8 \mathrm{~cm} ; \mathrm{G}=17 \%)$ at $\theta=0$ degrees. The contour of the projected breast is shown in green and the white lines correspond to the location of intensity profiles shown below.

Fig. 4: Horizontal (first column) andvertical (second column) intensity profiles of $S_{\text {hom }}^{\prime}(x, y)$ as illustrated in Figure 3(b). First and second rows correspond to a phantom of $\mathrm{T}=6 \mathrm{~cm}, \mathrm{R}$ $=12 \mathrm{~cm}$ and different glandularities $(2,17$ and 40\%) simulated at $\theta=0$ and $\theta=25$ degrees, respectively. Bottom row (e-f) represents phantoms of $\mathrm{R}=12 \mathrm{~cm}$ and thickness of $6 \mathrm{~cm}(\mathrm{G}$ $=17 \%)$ and $9 \mathrm{~cm}(\mathrm{G}=9 \%)$ for $\theta=0$ degrees. Vertical dashed lines represent the projected edge or boundary of the phantom.

Fig. 5: Relative error map, $\epsilon_{S}(\mathrm{x}, \mathrm{y})$, for several simulated breast models for 0 degree projection angles: (a) $\mathrm{T}=3 \mathrm{~cm}, \mathrm{R}=4 \mathrm{~cm}$ and $\mathrm{G}=35 \%$; (b) $\mathrm{T}=6 \mathrm{~cm}, \mathrm{R}=8 \mathrm{~cm}$ and $\mathrm{G}=17 \%$ and $(\mathrm{c}) \mathrm{T}=9 \mathrm{~cm}, \mathrm{R}=12 \mathrm{~cm}$ and $\mathrm{G}=9 \%$. Breast edge is highlighted with a solid black 
line.

Fig. 6: Relative error maps $\epsilon_{S}(\mathrm{x}, \mathrm{y})$ for a phantom with $\mathrm{T}=6 \mathrm{~cm}, \mathrm{R}=12 \mathrm{~cm}$ and $\theta=0$ degrees. Glandularities correspond to (a) 2, (b) 17 and (c) 40\%. Breast edge is highlighted with a solid black line.

Fig. 7: Relative error maps $\epsilon_{S}(\mathrm{x}, \mathrm{y})$ for a $6 \mathrm{~cm}$ thick and $\mathrm{R}=8 \mathrm{~cm}$ breast phantom $(\mathrm{G}=17 \%)$ at (a) 0, (b) 7.5 and (c) 25 degrees' projection angles. Breast edge is highlighted with a solid black line.

Fig. 8: Relative error maps $\epsilon_{S}(\mathrm{x}, \mathrm{y})$ for a $6 \mathrm{~cm}$ thick and $12 \mathrm{~cm}$ radius phantom $(\mathrm{G}=17 \%$, $\theta=0$ degrees), and different fibroglandular pattern distributions (P1, P2, P3). Breast edge is highlighted with a solid black line.

Fig. 9: $\epsilon_{S}(\mathrm{x}, \mathrm{y})$ using (a) $5 \mathrm{~cm}$ thick UPenn breast model $(\mathrm{G}=20 \%)$ and (b) $6 \mathrm{~cm}$ thick Duke University breast model $(\mathrm{G}=32 \%)$, acquired at $\theta=0$ degrees. Breast edge is highlighted with a solid black line. 